\title{
HIV: Support the patients, not the stigma
}

\section{The People Living with HIV Stigma Survey UK 2015: Stigmatising experiences and dental care}

Br Dent J 2018; 225: 143-150; http://dx.doi.org/10.1038/sj.bdj.2018.530

H I V. Three letters that, in the 1980 s, equated to a death sentence. HIV was a mysterious and rapidly fatal pandemic that sparked mass hysteria within society. In the decades since its discovery, there have been remarkable medical breakthroughs, most notably the development of antiretroviral drugs. Today, almost all patients living with HIV have an undetectable viral load, meaning that they are very unlikely to transmit the virus and won't experience any change in life expectancy. However, this is only true once HIV is detected and treated appropriately. Unfortunately, stigma and discrimination have followed the virus into the present day, which is driving a culture of silence and could be the greatest issue related to the disease.

Imagine making a dental appointment only to see your dentist 'double glove', to hear members of the dental team making negative comments about your health, or only being offered the last appointment of the day. Well, these were some of the astonishing outdated practices experienced by patients in the 2015 People Living with HIV Stigma Survey. Worryingly, over $90 \%$ of the respondents had avoided dental care, and this was more likely in those who were concerned that their diagnosis would result in them being refused treatment, treated differently or having their treatment delayed. As dental professionals we know the devastating impacts that self-exclusion from dental care can have on oral and general health, especially for those patients with chronic health issues. So why are we allowing these fears to persist?

Only half of the survey patients had informed their dental practice of their HIV status, and of those patients, a third felt unsupported by the dental team in relation to their HIV diagnosis. The researchers found this to be more common in those from ethnic minority backgrounds, in those who are transgender and in patients with financial issues. This highlights how HIV stigma within the dental practice can also relate to other patient factors, and again can encourage self-exclusion. Although

\section{Author Q\&A}

with Janine Doughty and Alastair Hudson From the People Living with HIV Stigma Index UK 2015 Advisory Group

\section{What made you choose to research HIV stigma?}

The People Living with HIV Stigma Survey UK 2015 built upon the work of The People Living with HIV Stigma Index in 2009, which championed a model of community-led research. To date much of the evidence gathered had been anecdotal. Our research team was motivated by the need to scientifically capture the lived experiences of the people from across the UK and Northern Ireland to generate a robust evidence base that could meaningfully affect change in the era of Undetectable $=$ Untransmittable $(\mathrm{U}=\mathrm{U})$. We know that in light of treatment advances the life expectancy for people living

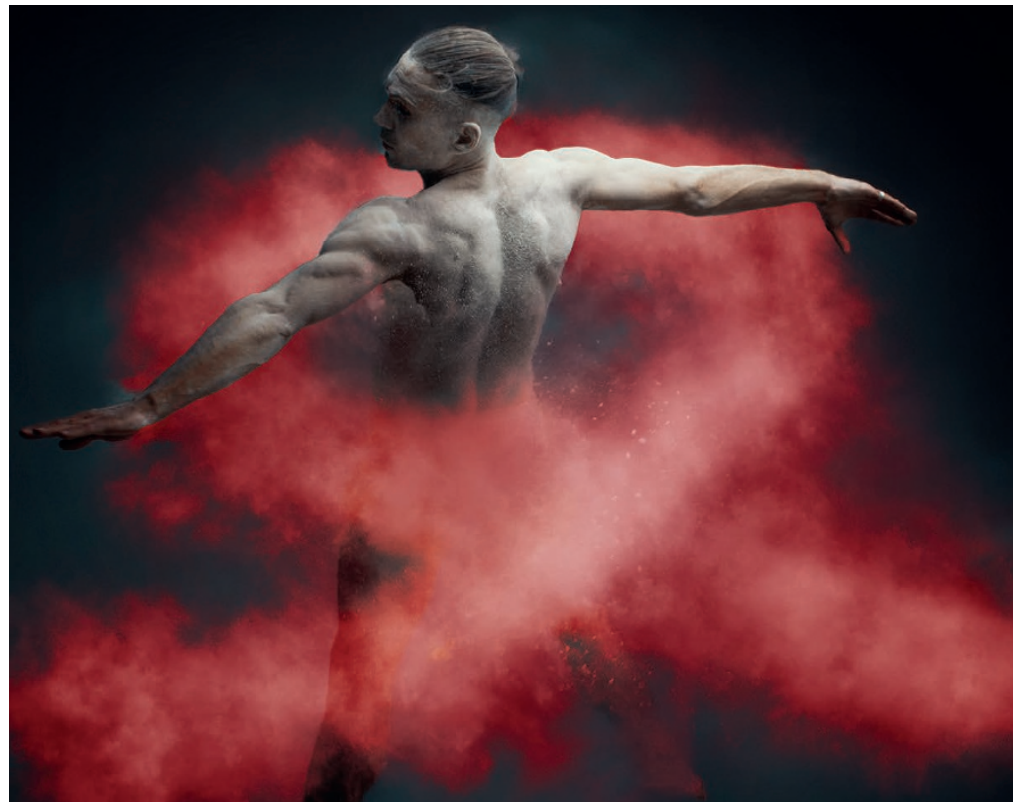

similar results were seen within general medical practice, patients felt less stigmatised when attending GUM clinics; a promising result that shows progression amongst medical colleagues.

The take-home message from this paper is that dental professionals must begin to have an awareness of the stigma and discrimination that HIV patients are facing. By doing so, we can learn how to take a non-discriminatory approach to HIV patients, and start supporting them appropriately. After all, the medicines to treat HIV have evolved, so why can't we?

By Roma McNeil

Dental Student, University of Leeds

with diagnosed HIV has increased to be comparable to that of HIVuninfected individuals. However, stigma and discrimination still significantly affect engagement with healthcare, treatment adherence, and overall health and wellbeing. We hope this data will stimulate discussion and impact on programme development, advocacy aims and policy change to really make a difference.

\section{What do the results of your study mean for the dental team?}

Despite the medical advances described above, people living with HIV still experience stigma when attending the dentist; as a result some patients choose not to make their dentist aware of their HIV status or avoid attending the dentist altogether. Stigmatising practice includes, but is not limited to, using excessive barrier protection such as doublegloving, limiting appointments to the end of a treatment session, treating people living with HIV differently - for example, by refusing or delaying treatment. This study highlights the need for the dental team to update their HIV knowledge, practice universal cross infection control for all patients, and develop culturally sensitive awareness in order to eliminate experiences of HIV stigma in the dental setting. 